guidelines; B. Evaluation of local to national level government early years sector health eating guidelines using updated CWT guidelines as the "gold standard"

Main Outcome Measures Identification of new evidence to augment CWT 2006 guidelines. Identification of gaps in early years sector health eating guidelines at local to national levels.

Results Seven studies were deemed appropriate for analysis alongside the CWT eat well guidelines. Ten key recommendations and sub-recommendations were identified in relation to promoting healthy eating in the early years setting: role of government; nursery policy/guidelines; training; information and communication; menu planning; parents; atmosphere and encouragement; learning through food; sustainability; and equal opportunities. The evaluation of the seven government guidelines revealed that they had all included the ten key recommendations but there was sporadic cover of sub-key recommendations and in several cases detail was limited.

Conclusions The CWT guidelines for healthy eating in children under five remain highly appropriate. However, further work needs to be done to understand the optimal content and detail of such guidelines to maximise their effectiveness. Guidelines are only one element of what should be an integrated approach to implementing healthy eating across the early years sector. All key and sub-key CWT recommendations should be included in government guidelines. Guidance on the presentation of food and practical tips to encourage children to eat their food needs to be included, especially at local level. The wider role that food and meal times can play in children achieving Early Years Foundation Stage competencies should be explored. And crucially, the possible ways that nurseries can support parents to achieve healthy eating within the home.

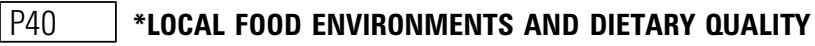

doi:10.1136/jech.2010.120477.40

${ }^{1} \mathrm{R}$ Layte, ${ }^{2} \mathrm{~J}$ Harrington, ${ }^{1} E$ Sexton, ${ }^{2} \mathrm{~J}$ Perry, ${ }^{1} \mathrm{~S}$ Lyons. ${ }^{1}$ Economic and Social Research Institute, Dublin, Ireland; ${ }^{2}$ Department of Epidemiology and Public Health, University College Cork, Ireland

Introduction Dietary quality is an important predictor of health outcome and plays a prominent role in premature death from a number of chronic health conditions including cardiovascular disease and some cancers. Socio-economic gradients in food consumption are observed worldwide. Inadequate nutritional intake and poor dietary habits are also associated with food poverty, a well documented global public health problem, which is a complex and multi-faceted problem, with widespread consequences for dietary intake. Many definitions of food poverty have appeared in the literature, the majority of which include, to a more or lesser extent, the issues of food affordability, access to and availability of a healthy and nutritious diet to be consumed in a socially and acceptable way. Much of the published literature on the associations between access to and availability of healthy diets focuses on the food environments and individual dietary components for example, fruit and vegetables. However, due to the complex nature of the determinants of dietary habits, measuring dietary quality using an overall dietary index may provide additional insight. The physical availability of food has been shown to be a significant predictor of dietary quality.

Objectives This paper investigates the influence of the economic and physical availability of food on individual dietary quality.

Methods Data are drawn from a two-stage clustered sample of 10364 individuals aged 18+ from the Republic of Ireland. Diet is assessed via a food frequency questionnaire and the results scored in terms of cardiovascular risk. Food availability is measured in terms of distance to and density of different types of food outlets. Dietary quality is decomposed using fixed effect multi-level regression models.
Results More socio-economically advantaged individuals are likely to live closer to a larger food store and to consume a better diet. Controlling for individual and household characteristics, individuals who live closer to a larger food outlet or who live in an area with a higher density of larger food outlets have a significantly better diet. Conclusions There are significant and pronounced socio-economic gradients in diet and nutrition in the Republic of Ireland that may contribute to health inequalities. Food availability may also be a significant contributor to poorer dietary quality with the result that poorer households in poor areas are doubly disadvantaged.

*High scoring abstract.

\section{P41 YOUNG CHILDREN'S FOOD IN DAY CARE SETTINGS: A QUALITATIVE STUDY OF PRESCHOOL NUTRITION POLICY AND PRACTICE}

doi:10.1136/jech.2010.120477.41

K S Bristow, S Capewell, F Lloyd-Williams. Public Health, University of Liverpool, Liverpool, UK

Objectives To explore nutrition in local day care settings in order to develop a package of interventions that will promote healthy eating in Liverpool across deprived communities.

Design Qualitative-participant observation, direct observation, interviews.

Setting Community-preschool nurseries.

Participants Nursery managers, cooks, staff, parents, children (total=36).

Main Outcome Measures Up to date assessments of food policy and provision in a range of nursery settings highlighting inequalities in good practice and gaps in existing evidence. Identification of specific needs and barriers in nurseries serving areas of deprivation.

Results Nurseries have a potentially important role in supporting parents in their children's and their own healthy eating. Level and depth of communication between the nursery and parents is important regarding what children have eaten both at home and at the nursery. Private nurseries have minimal access to information and guidelines compared to those based in Sure Start children's centres. Most nurseries do not have a specific healthy eating policy but use their menu planning as the way to maintain a focus on healthy eating. Most nurseries have an appropriately balanced $4-$ week menu plan. Use of gravy and pre-prepared sauces is problematic and needs attention. Cooks' level of knowledge, experience and motivation is important. None had been trained in healthy eating for under fives. Meal times can be an important means of developing social skills and achieving Early Years Foundation Stage competencies. Making meal times fun and appropriate-sized cutlery and crockery are important to encourage children to eat. Communication between different levels of government and departments needs strengthening. Additional funding for training will be, essential to promote menu planning and cooking, but also for encouraging healthy eating and learning through food.

Conclusions Nurseries are genuinely interested in implementing healthy eating policies and need further support to achieve this. Support should include: improved mechanisms for effective communication between all government levels as well as with nurseries; funded training for cooks and managers in menu planning, cost effective food sourcing, and food preparation. Classroom staff should receive training in strategies to encourage healthy eating habits and how to facilitate food related learning and social skills development. Nurseries appear to have a key role in working with parents to encourage healthy eating at home. Further research is needed to understand the ways in which nurseries can be supported to achieve this. 